\title{
A Child Diagnosed With Treatment-Resistant Polyarteritis Nodosa: Can the Clinical Diagnosis be Different?
}

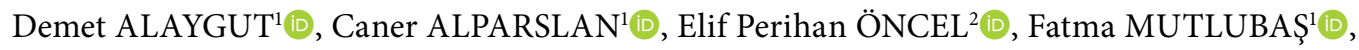 \\ Tunç ÖZDEMİR ${ }^{3}$, Önder YAVAŞCAN ${ }^{1} \mathbb{D}$, Belde KASAP DEMİR ${ }^{4}$ (]) \\ ${ }^{1}$ Department of Pediatric Nephrology, Tepecik Training and Research Hospital, Izmir, Turkey \\ ${ }^{2}$ Department of Pediatrics, Tepecik Training and Research Hospital, Izmir, Turkey \\ ${ }^{3}$ Department of Pediatric Surgery, Tepecik Training and Research Hospital, Izmir, Turkey \\ ${ }^{4}$ Department of Pediatric Nephrology, Katip Çelebi University Faculty of Medicine, Izmir, Turkey
}

\begin{abstract}
Polyarteritis nodosa (PAN) is a necrotizing systemic vasculitis involving the wall of small and medium sized arteries. The histological aspect is defined by the presence of fibrinoid necrosis and an infiltrate rich in neutrophil polynuclears in the artery wall and rare granulomas. CECR1 (Cat Eye Syndrome Chromosome Region 1) gene mutation causing adenosine deaminase 2 (ADA2) deficiency is also associated with systemic inflammation, vasculopathy, and frequently PAN. Strokes, neurological involvement, and gastrointestinal involvement have poor prognosis in the cases with ADA2 deficiency particularly in early stage. In this article, we report a 17-year-old male patient diagnosed with PAN who had severe gastrointestinal system involvement that was resistant to intensive and conventional immunosuppressive treatment and showed a fatal course despite the emergency surgical intervention. After the patient was exitus, he was detected to have a heterozygous mutation (V276A) of familial Mediterranean fever (FMF) and also a homozygous ADA2 mutation. The aim of this article is to highlight that ADA2 deficiency may be present in treatment-resistant PAN cases who apply due to severe systemic involvement. In this case, accompanying FMF mutation was also observed.

Keywords: Adenosine deaminase 2 deficiency; adolescent; familial Mediterranean fever; polyarteritis nodosa.
\end{abstract}

Adenosine deaminase 2 (ADA2) deficiency is a cause of vasculopathy that is coded by the CECR1 (Cat Eye Syndrome Chromosome Region 1) gene and that causes overlapping with polyarteritis nodosa (PAN) as a result of recessive mutations. ADA2 is both a major extracellular adenosine deaminase and an adenosine deaminase-related growth factor. In humans, the irreversible degradation of adenosine to inosine and deoxyadenosine to deoxyinosine is catalyzed by intracellular ADA1 and extracellular ADA2. ${ }^{1}$ Recessive ADA1 mutations are a well-known cause of severe combined immune deficiency.
However, ADA2 deficiency manifests as increased vascular inflammation without clinically apparent immune deficiency. This vasculopathy is characterized by highly varied age at onset, severity, and organ involvement, even within families and among patients with the same mutations. Manifestations range from severe or fatal systemic vasculitis or multiple strokes to limited cutaneous manifestations in children. ${ }^{2}$ This disease encloses a wide spectrum of vascular and inflammatory manifestations, including earlyonset strokes, recurrent fevers, and systemic vasculopathy, which is often consistent with PAN. ${ }^{3}$

Received: June 22, 2018 Accepted: December 11, 2018 Published online: April 22, 2019

Correspondence: Demet Alaygut, MD. Tepecik Eğitim ve Araştırma Hastanesi Pediatrik Nefroloji Kliniği, 35180 Konak, İzmir, Turkey. Tel: +90 232 - 4693799 e-mail: alaygutdemet@gmail.com 
Most cases are hardly differentiated from PAN with not only their clinical findings but also their histopathological characteristics. Affected children have varied presentations that are not sufficient for clinical diagnosis at early age. In addition, constitutional symptoms also complicate differentiation. This article reports a patient meeting the PAN diagnostic criteria accompanied with severe treatment-resistant and fatal-course ADA2 deficiency and FMF.

\section{CASE REPORT}

A 17-year-old male patient presented to the emergency department for evaluation of severe abdominal pain of approximately two months duration. His past history revealed that an abdominopelvic ultrasonography and a computed tomography angiography were performed before he was referred. They showed aneurysmal changes in all abdominal veins (Figure 1). Physical examination was notable for a blood pressure of $200 / 100 \mathrm{mmHg}$, paleness, myalgia in the legs and arms, and abdominal sensitivity. His weight and height were $28 \mathrm{~kg}$ (-3.7 standard deviation [SD]) and $149 \mathrm{~cm}(-3.89 \mathrm{SD})$, respectively. Laboratory evaluation revealed anemia (hemoglobin $9.6 \mathrm{~g} / \mathrm{dL}$, mean corpuscular volume: $64.3 \mathrm{fL}$ ) and elevated acute phase reactants (erythrocyte sedimentation rate: $82 \mathrm{~mm} /$ hour, C-reactive protein: $205.2 \mathrm{mg} / \mathrm{dL}$ ). Urinary analysis showed

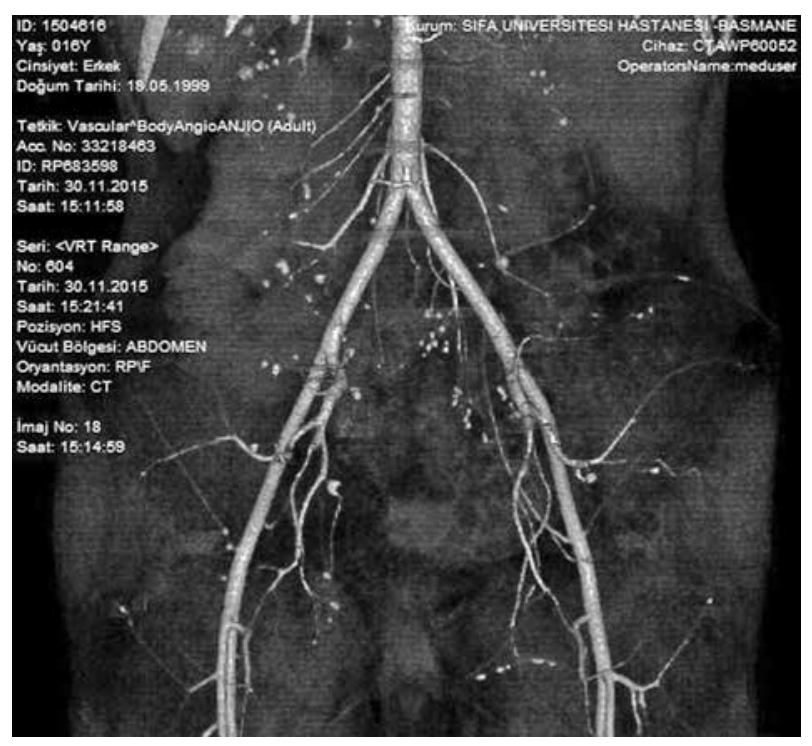

Figure 1. Aneurysmal changes in all abdominal veins.
+3 proteinuria. Complement components (C3, C4), antistreptolysin O, viral serologies, perinuclear antineutrophil cytoplasmic antibodies, and antineutrophil cytoplasmic antibody were observed as negative. The patient was accepted as PAN due to the imaging results, weight loss, myalgia, and renal involvement. For blood pressure elevation; sodium nitroprusside infusion was applied at first and then calcium channel blocker and angiotensin-converting-enzyme inhibitor were administered. During follow-up, he had a suspect seizure attack and complained of temporary loss of vision. A written informed consent was obtained from the patient.

Cranial magnetic resonance imaging (MRI) and diffusion imaging were normal. Regarding the diagnosis of PAN, administration of pulse methylprednisolone (PMP) was planned at a dose of $30 \mathrm{mg} / \mathrm{kg}$ and $500 \mathrm{mg} / \mathrm{m}^{2} /$ day intravenous (IV) cyclophosphamide treatment. Due to insufficient oral intake and continuous stomach pain, total parenteral nutrition was started. Additional PMP treatments were added for the case from whom partial clinical and laboratory response were obtained in the follow-up. After nine doses of PMP and two IV cyclophosphamide treatments,

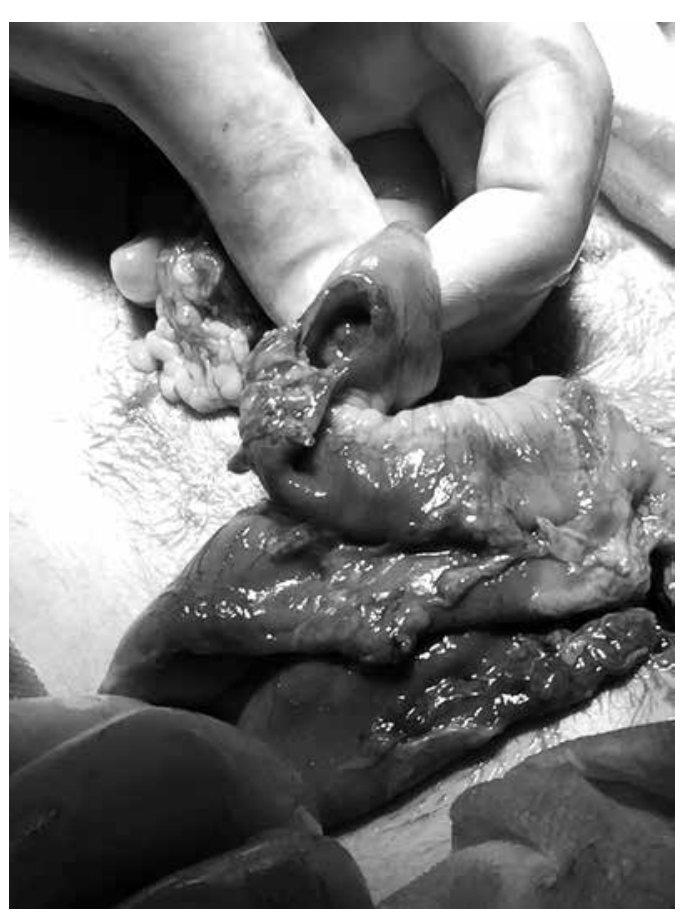

Figure 2. Total ileum and column resection were applied and jejunostomy was performed. 


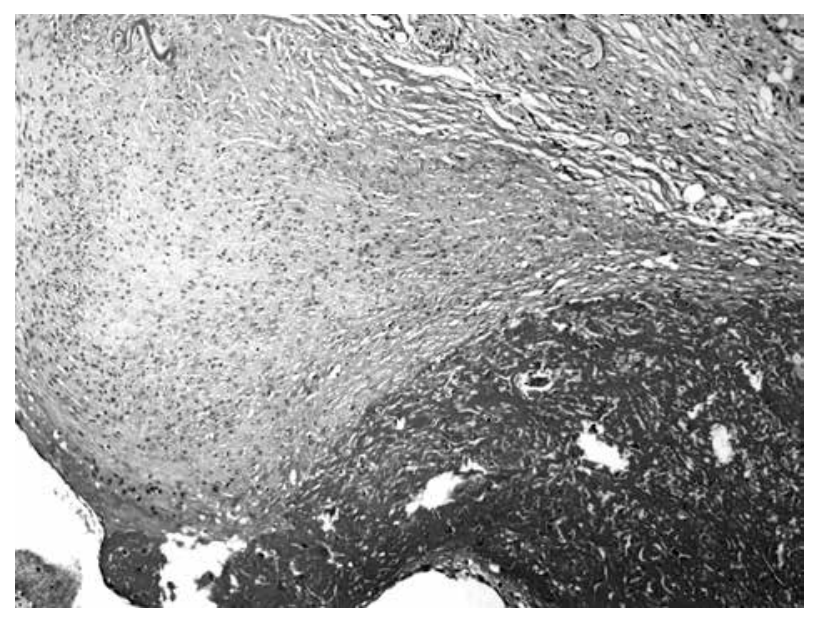

Figure 3. Pathological examination indicated fibrinoid necrosis in small and middle veins that was compatible with polyarteritis nodosa.

administration of infliximab, a tumor necrosis factor-alpha (TNF- $\alpha$ ) blocker, was planned. In the first month of follow-up, the patient, who had aggravated stomach pain, was assessed as intestinal perforation. Total ileum and column resection were applied and jejunostomy was performed (Figure 2). Pathological examination indicated fibrinoid necrosis in small and middle veins that was compatible with PAN (Figure 3). The patient was taken to intensive care unit with postoperative sepsis and a new perforation that was observed in the second exploration. He was exitus on the $44^{\text {th }}$ follow-up day. Genetic studies showed V726A heterozygous mutation in Mediterranean fever (MEFV) gene and p.G47A (c.140 G>C homozygous mutation in CECR1 gene).

\section{DISCUSSION}

Deficiency of ADA 2 is a monogenic autoinflammatory disorder, described in 2014 by two international study groups simultaneously. ${ }^{2,4}$ Examination of patients' demographic and clinical features in the literature revealed that many had a previous diagnosis of PAN. Because clinical features mainly depend on the affected small and medium-sized arteries these two clincs are interfere.

Polyarteritis nodosa is a systemic necrotizing and rarely seen vasculitis particularly affecting medium and small-diameter arteries. ${ }^{5}$ Clinical spectrum of idiopathic PAN has quite different types, varying from a single organ involvement to polyvisceral involvement. ${ }^{5,7}$ Occlusion or rupture of inflamed arteries causes ischemia or hemorrhage in the organs affected. ${ }^{5}$ Nonspecific constitutional symptoms (fatigue, loss of weight, fever, arthralgia, and myalgia etc.) and all the results associated with the dysfunction or damage of the target organ are seen in patients. ${ }^{6}$ Clinically, PAN comes to mind with constitutional symptoms and multisystemic involvement. ${ }^{7}$ Diagnostic criteria of PAN include one of the histopathological or angiographic abnormalities and at least one skin involvement, myalgia or muscle tenderness, hypertension, peripheral neuropathy, and renal involvement. ${ }^{7}$ Our patient was diagnosed with PAN associated with angiography findings, constitutional symptoms, myalgia, muscle tenderness, hypertension, and renal involvement. In a retrospective study evaluating 69 pediatric patients, patients were followed-up for 32 years and cutaneous involvement, fever, myalgia, arthritis/arthralgia, and weight loss were assessed as the most frequent initial symptoms. ${ }^{8}$

Gastrointestinal system and kidneys are frequently affected. Gastrointestinal involvement ratio was specified as $44 \%$ and observed as an acute abdominal finding in one third of the cases. .10 Gastrointestinal hemorrhage and infarction are generally observed while perforation is rare. Perforation may occur in both small and large intestines and progress fatally. ${ }^{11}$ It was reported that intestinal perforation can be the first result of PAN both in pediatric and adult age groups. ${ }^{11,12}$ In our case, gastrointestinal perforation was not the first finding at the time of diagnosis but it occurred twice in follow-up despite intensive immunosuppressive treatment. In the samples taken surgically, the presence of fibrinoid necrosis supported the gastrointestinal involvement. PAN cases were reported to occur with atypical lifethreatening acute abdominal picture and mostly in the form of a current mesenteric ischemia. ${ }^{13}$ Although mesenteric ischemia is one of the rare causes of intestinal infarction, $38.3 \%$ of cases with PAN applied with gastrointestinal findings and $42.9 \%$ of these underwent emergency surgical laparotomy with high mortality ratio. ${ }^{13}$ When we analyzed our case for FMF gene, we detected the presence of heterozygous mutation (V276A). 
FMF is the most frequent autoinflammatory disease with autosomal recessive transition and it accompanies some inflammatory diseases such as systemic vasculitis. ${ }^{14}$ In patients having FMF associated with PAN, FMF symptoms develop during the years before the occurrence of PAN symptoms. ${ }^{15}$ In our case, intermittent agonizing stomach aches within last two years were the possible findings of FMF. A total of 38\% MEFV mutations accompanied to 29 pediatric patients diagnosed with PAN in Turkey. ${ }^{16}$ It was challenging to establish a differential diagnosis for our patient since there were many intertwined clinical symptoms, of which many may be seen in FMF, PAN, or ADA2 deficiencies.

However, central nervous system involvement is a frequent manifestation of this rare disease and it should primarily suggest a deficiency of ADA2 in the differential diagnosis of patients already known to have PAN. Neurological findings encompass transient ischemic attack, ischemic and hemorrhagic strokes, and a wide range of severe manifestations. ${ }^{17}$ Although the cranial MRI of our patient was normal, we had to consider non-PAN diagnoses because of the suspect seizures and temporary visual loss.

In patients with vasculopathy and PAN, we may suspect ADA2 deficiency and loss-of-function mutations in CECR1 causing ADA2 deficiency. ${ }^{18}$ In the first reports related to ADA2 deficiency, positive family history, livedo reticularis, early-onset stroke, and peripheral nervous system involvement were defined as well as immunodeficiency in some patients. ${ }^{18}$ Nanthapisal et al. $^{18}$ assessed the CECR1 mutation of 15 symptomatic cases and determined homozygous or compound heterozygous mutation in all cases. When phenotypic findings of the cases were examined, livedo reticularis was present in 73.3\%, neurological involvement in 53.3\%, and immunodeficiency in $46.7 \%$. Mutation was determined in five asymptomatic cases (relatives of index cases). As a result, authors recommended to perform CECR1 screening in the unaffected siblings of index cases, familial vasculitis cases, and standard treatment-resistant PAN cases. ${ }^{18} \mathrm{We}$ determined a homozygous mutation in the CECR1 p.G47A (c.140 G>C) gene, which we examined since our patient had severe gastrointestinal involvement and did not respond to standard immunosuppressive treatment.
Further studies have been conducted in recent years on ADA2 deficiency occurring in different phenotypes and mimicking the PAN symptoms, emphasizing its pathogenesis and phenotypegenotype relations. ADA2 deficiency should definitely be investigated in suspicious cases.

In February 2014, two independent studies, one held by the American National Institutes of Health in Bethesda. ${ }^{4,19}$ and the other one by the Israeli Shaare Zedek Medical Center in Jerusalem, ${ }^{2}$ identified this new clinical entity, named "The deficiency of ADA2, often familial, characterized by early onset livedoid rash associated with systemic inflammation (fever and elevation of acute phase reactants). Some patients presented with ischemic or hemorrhagic cerebral stroke and others with vasculopathy-related manifestations (hypertension, gastrointestinal symptoms), hepatosplenomegaly, peripheral neuropathy, and mild immunodeficiency. In many cases, both the clinical manifestations and histological findings were consistent with the diagnosis of PAN.

Unfortunately, we obtained the result for the genetic analysis (both for FMF and ADA2) of our patient after he was exitus. Because the case was highly compatible with classical PAN together with initial clinical and laboratory results, high dose steroid and cyclophosphamide treatment was started. However, due to failure to receive any clinical or laboratory response, repeated doses (totally nine doses) of steroids had to be continued. Afterwards, we associated this condition with the steroid resistance defined in ADA2 deficiency cases in the literature. Similarly, drugs such as cyclophosphamide, azathioprine, and methotrexate were also reported to be ineffective. ${ }^{19}$ Navon Elkan et al. ${ }^{2}$ reported that they received complete response in eight of 10 cases who were administered anti-TNF drugs (etanercept, adalimumab, and infliximab). Unfortunately, we could not administer anti-TNF- $\alpha$ drug in our patient since he was being followed-up for sepsis in the intensive care unit.

In addition to anti-TNF- $\alpha$ therapy, ADA2 enzyme replacement therapy by fresh-frozen plasma (FFP) has provided substantial beneficial effects in some patients as reported by Sahin et al. ${ }^{17}$ who administered monthly FFP infusions after the diagnosis of ADA2 deficiency in three patients 
in their case series. Furthermore, a possible role of hematopoietic stem cell transplantation has been postulated to be effective by Zhou et al., ${ }^{4}$ and Navon Elkan et al., ${ }^{2}$ by providing ADA2 producing monocytes and therefore normalizing the plasmatic levels of the enzyme.

In conclusion, ADA2 deficiency is a genetic condition associated with inflammatory vasculopathies such as PAN. Age of disease onset, disease findings, and clinical courses may be quite variable. ADA2 deficiency should be kept in mind in patients diagnosed with PAN or cutaneous PAN and/or in case of severe organ involvement. Genetic tests should be evaluated in the presence of positive family history. Diagnosis of the disease is compulsory to provide effective treatment and perform family screenings.

\section{Declaration of conflicting interests}

The authors declared no conflicts of interest with respect to the authorship and/or publication of this article.

\section{Funding}

The authors received no financial support for the research and/or authorship of this article.

\section{REFERENCES}

1. Gaspar HB, Aiuti A, Porta F, Candotti F, Hershfield MS, Notarangelo LD. How I treat ADA deficiency. Blood 2009;114:3524-32.

2. Navon Elkan P, Pierce SB, Segel R, Walsh T, Barash $\mathrm{J}$, Padeh $\mathrm{S}$, et al. Mutant adenosine deaminase 2 in a polyarteritis nodosa vasculopathy. $\mathrm{N}$ Engl $\mathrm{J}$ Med 2014;370:921-31.

3. Garg N, Kasapcopur O, Foster J, Barut K, Tekin A, Kızılkılıç $\mathrm{O}$, et al. Novel adenosine deaminase 2 mutations in a child with a fatal vasculopathy. Eur $\mathrm{J}$ Pediatr 2014;173:827-30.

4. Zhou Q, Yang D, Ombrello AK, Zavialov AV, Toro C, Zavialov AV, et al. Early-onset stroke and vasculopathy associated with mutations in ADA2. N Engl J Med 2014;370:911-20.

5. De Virgilio A, Greco A, Magliulo G, Gallo A, Ruoppolo G, Conte M, et al. Polyarteritis nodosa: A contemporary overview. Autoimmun Rev 2016;15:564-70.
6. Lhote F, Cohen P, Guillevin L. Polyarteritis nodosa, microscopic polyangiitis and Churg-Strauss syndrome. Lupus 1998;7:238-58.

7. Barut K, Sahin S, Kasapcopur O. Pediatric vasculitis. Curr Opin Rheumatol 2016;28:29-38.

8. Eleftheriou D, Dillon MJ, Tullus K, Marks SD, Pilkington CA, Roebuck DJ, et al. Systemic polyarteritis nodosa in the young: a single-center experience over thirtytwo years. Arthritis Rheum 2013;65:2476-85.

9. Stone JH. Polyarteritis nodosa. JAMA 2002;288:1632-9.

10. Buldukoglu OC, Koklu S, Koklu H, Uluoglu O, Kulduk G. Intestinal perforation as the initial presentation of polyarteritis nodosa in an older adult. Geriatr Gerontol Int 2015;15:121-2.

11. Gündoğdu HZ, Kale G, Tanyel FC, Büyükpamukçu $\mathrm{N}$, Hiçsönmez A. Intestinal perforation as an initial presentation of polyarteritis nodosa in an 8-year-old boy. J Pediatr Surg 1993;28:632-4.

12. Bağci P, Erdamar S, Erzin Y, Karataş A, Tuncer M. A case of polyarteritis nodosa diagnosed after recurrent, multiple intestinal perforations. Turk J Gastroenterol 2009;20:71-2.

13. Asti E, Pogliani L, Tritella S, Bonavina L. Polyarteritis nodosa and acute abdomen: A role for laparoscopy? Int J Surg Case Rep 2015;17:161-3.

14. Ozen S. Mutations/polymorphisms in a monogenetic autoinflammatory disease may be susceptibility markers for certain rheumatic diseases: lessons from the bedside for the benchside. Clin Exp Rheumatol 2009;27:29-31.

15. Ozen S, Ben-Chetrit E, Bakkaloglu A, Gur H, Tinaztepe K, Calguneri M, et al. Polyarteritis nodosa in patients with Familial Mediterranean Fever (FMF): a concomitant disease or a feature of FMF? Semin Arthritis Rheum 2001;30:281-7.

16. Yalçinkaya F, Ozçakar ZB, Kasapçopur O, Oztürk A, Akar N, Bakkaloğlu A, et al. Prevalence of the MEFV gene mutations in childhood polyarteritis nodosa. $\mathrm{J}$ Pediatr 2007;151:675-8.

17. Sahin S, Adrovic A, Barut K, Ugurlu S, Turanli $\mathrm{ET}$, Ozdogan $\mathrm{H}$, et al. Clinical, imaging and genotypical features of three deceased and five surviving cases with ADA2 deficiency. Rheumatol Int 2018;38:129-36.

18. Nanthapisal S, Murphy C, Omoyinmi E, Hong Y, Standing A, Berg S, et al. Deficiency of Adenosine Deaminase Type 2: A Description of Phenotype and Genotype in Fifteen Cases. Arthritis Rheumatol 2016;68:2314-22.

19. Caorsi R, Penco F, Schena F, Gattorno M. Monogenic polyarteritis: the lesson of ADA2 deficiency. Pediatr Rheumatol Online J 2016;14:51. 